\title{
Low Salt Diet in Pregnant Mothers Is Associated with Enhanced Salt Appetite in Their Offspring of Dahl Salt-Sensitive Rats
}

\author{
Anna Hara, Ranna Chow, Dong Dong Du, Hiroe Sakuyama, Yoshio Uehara \\ Division of Clinical Nutrition, Faculty of Home Economics, Kyoritsu Women's University, Tokyo, Japan \\ Email: yuehara@kyoritsu-wu.ac.jp
}

Received 25 July 2014; revised 20 August 2014; accepted 5 September 2014

Copyright (C) 2014 by authors and Scientific Research Publishing Inc.

This work is licensed under the Creative Commons Attribution International License (CC BY). http://creativecommons.org/licenses/by/4.0/

(c) (i) Open Access

\section{Abstract}

Objectives: We investigated an influence of salt intake during gestation or lactation in salt preference of weaning Dahl salt sensitive (Dahl S) strain. Material and Methods: Nine-week-old female Dahl $S$ rats, after mated with the male, were divided into 1) high-salt group fed a $4 \% \mathrm{NaCl}$ diet (high-salt mother) or 2) low-salt group fed a $0.3 \% \mathrm{NaCl}$ diet (low-salt mother) during gestation or lactation periods. Using $0.4 \%, 0.6 \%$ and $0.8 \%(w / v)$ saline solutions, we assessed salt preference in their offspring after weaning. Systolic blood pressure (SBP) was determined by tail cuff method. Results: Both the male and female offspring from low-salt mothers during gestation consumed equal amounts of any saline solution. However, the amount of each saline solution was higher in the offspring of low-salt mothers than those of high-salt mothers. This resulted in a significant increase of salt intake in both the male and female offspring of low-salt mothers compared with those of high-salt mothers. In contrast, both the male and female offspring from low-mothers during lactation rather preferred the lower concentration of saline solution, and this resulted in less salt intake in the offspring of low-salt mothers than those of high-salt mothers. Conclusions: We demonstrated that low salt diet in pregnant mothers increased salt intake of their offspring. The offspring of mothers fed a low salt diet during lactation had less salt than those of high-salt mothers. The influence of salt intake of mothers in their offspring varies along with the growth stage of their babies.

\section{Keywords}

Salt Appetite, Neonate, Salt-Sensitive Hypertension, Dahl Rat, Programming, Gestation, Lactation

\section{Introduction}

In Japan, more than 35 million people have suffered from hypertension and approximately $50 \%$ of them are be-

How to cite this paper: Hara, A., Chow, R., Du, D.D., Sakuyama, H. and Uehara, Y. (2014) Low Salt Diet in Pregnant Mothers Is Associated with Enhanced Salt Appetite in Their Offspring of Dahl Salt-Sensitive Rats. Food and Nutrition Sciences, 5, 1904-1913. http://dx.doi.org/10.4236/fns.2014.519202 
lieved to be salt-sensitive. Since hypertension is the leading risk factor for various organ damages, in order to prevent the onset of hypertension, salt restriction has been campaigned over decades. However, unfortunately, the role of salt intake for health has not fully discussed depending on their life-stage.

Over several decades much effort has been made to disclose the mechanism of hypertension in human. Various cardiovascular regulation systems, i.e. renin-angiotensin-aldosterone system, endothelium-dependent vasodilators, adrenergic nervous system or gene-mediated mechanisms, have been investigated. Among them, the theme, salt and hypertension, has been interested so much for a long time.

Interestingly, recent studies have highlighted the role of life-style of mothers during gestation in the development of hypertension and metabolism-related diseases in adulthood. In fact, some investigators have reported that premature babies likely increase risk of hypertension and cardiovascular diseases in adulthood [1]-[3]. Moreover, a very low birth is associated with higher indexes of insulin resistance and glucose intolerance as well as hypertension [4] [5]. Salt intake of mothers is linked to blood pressure elevation or cardiovascular modeling in adulthood [6]-[8]. Crystal and Bernstein reported that mother's morning sickness and a decrease in body fluid may responsible for infant salt preference [9]. These studies strongly suggested that the food behavior or diseases in adulthood might be programmed during growth in mother's uterus.

However, the knowledge is not enough about the implications of food behavior of mothers during gestation or lactation in food preference and related diseases of their offspring. In this sense, studies on this field are very interesting to understand the development of salt preference and salt-related diseases in their youth or adulthood. If the hypothesis that salt preference is programmed during gestation or lactation of mothers is true, we may obtain a new strategy to prevent elevation of blood pressure and related diseases. In the present study, we attempted to reveal whether or how salt intake of mothers during gestation or lactation affects salt preference of their offspring.

\section{Materials and Methods}

\subsection{Design of Experiment}

\subsection{1. (EXP-1) Salt Preference of Baby Rats from Mothers Fed Low or High Salt Diet during Gestation} We purchased 4-week-old male and female Dahl salt-sensitive (Dahl S/Iwai) rats from Sankyo Laboratories, Inc., Tokyo, Japan. The Dahl S rats were fed a regular chow $(0.75 \% \mathrm{NaCl}$ diet) (CEL Rodent Diet CE-7, CLEA Japan, Inc., Tokyo, Japan) and at 8 weeks old the Dahl rats were mated for 1 week. The female rats mated were separated to each cage, and divided randomly into 2 groups as follows: 1) 4 Dahl S rats fed a low-salt (0.3\% $\mathrm{NaCl}$ ) diet (F2Dahl-0.3\%, Oriental Yeast Co., Ltd., Tokyo, Japan) (0.3\% DS) and 2) 4 Dahl S rats fed a highsalt (4\% NaCl) diet (F2Dahl-4\%, Oriental Yeast) (4\% DS) (Figure 1). Water was available ad libitum.

Three weeks after the matching, the mothers began to produce infants. The mothers were fed the regular diet during lactation. The weaning baby rats were put on the regular chow until and during the salt preference test. During the salt preference test, water was changed to three different concentrations of saline solutions.

\subsection{2. (EXP-2) Salt Preference of Baby Rats from Mothers Fed Low or High Salt Diet during Lactation}

We investigated an influence of salt intake of mothers during lactation in the salt preference of their weaning baby rats (Figure 1). Briefly, Dahl S rats were fed the regular chow during the mating and subsequent gestation. Immediately after babies were born, the mothers were divided randomly into two groups: 1) 4 mothers fed the low-salt diet and 2) 4 mothers fed the high salt diet during lactation. After weaning the baby rats were put on the regular chow and the salt preference was determined for 4 days as described. Water was available ad libitum.

\subsection{Assessment of a Salt Preference}

To determine the salt preference, we measured amounts of different concentrations of saline solutions consumed during 4-day test period. The rats to be tested had free access to any concentration of saline solutions. The position of bottles in a cage was randomly changed every day. The amounts of saline solutions consumed were measured every day for 4 days. From the amounts of saline solutions consumed, we calculated the salt intake per day in each rat.

We preliminarily examined salt intake of 7-week-old male Dahl S rats fed the regular chow on consecutive two days. On Day-1 $0.4 \%$ saline solution was preferred and on Day-2 there were no differences among the different 


$$
\text { Age (week) }
$$

male DS
$0-3$

4-5

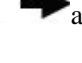

female DS

-1):

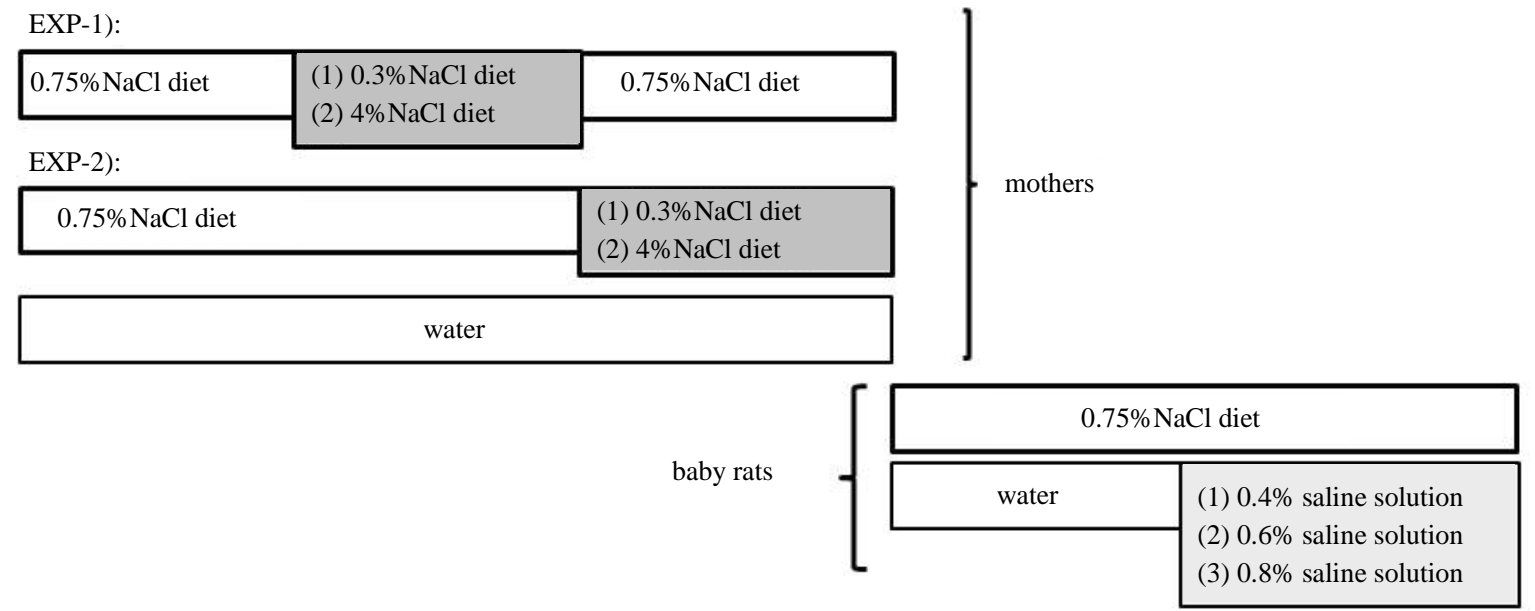

Figure 1. Protocol of salt preference test. This graph represents design of the study on salt preference of offspring of lowand high-salt mothers during gestation (EXP-1)) and during lactation (EXP-2)). Two weeks after weaning, the salt preference test was done. DS, Dahl salt sensitive rats.

concentrations of saline solution (Figure 2). In the present study, we utilized $0.4 \%, 0.6 \%$ and $0.8 \%(\mathrm{w} / \mathrm{v})$ saline solutions for the preference test mainly because this combination seemed sensitive to detect changes of saline solutions consumed. In addition, we attempted to minimize day-by-day variation by testing the preference for consecutive 4 days.

\subsection{Blood Pressure Determination}

Systolic blood pressure was determined by the tail-cuff method according to Friedman and Freed [10].

\subsection{Statistical Analysis}

All statistical analyses were performed using STATISTICA software (StatSoft, Tulsa, OK). Values were expressed as means \pm SD. Differences were assessed by one-way factorial analysis of variance (ANOVA) followed by post-hoc least significant difference (LSD) test. $P$-values less than 0.05 were considered statistically significant.

\subsection{Guidelines for Handling Rats for Experiments}

We followed the guidelines for experimental animal handling, and our study was approved by the Animal Care Committee of the Kyoritsu Women's University. The experiment was conducted in accordance with the National Institutes of Health (NIH) guidelines.

\section{Results}

\section{1. (EXP-1) Salt Preference of Baby Rats from Mothers of Different Salt Intake during Gestation}

The basal data of the weaning rats before the test were shown in Table 1. In both the male and female baby rats, the body weight was less in the offspring from low-salt mothers than those from high-salt mothers during 


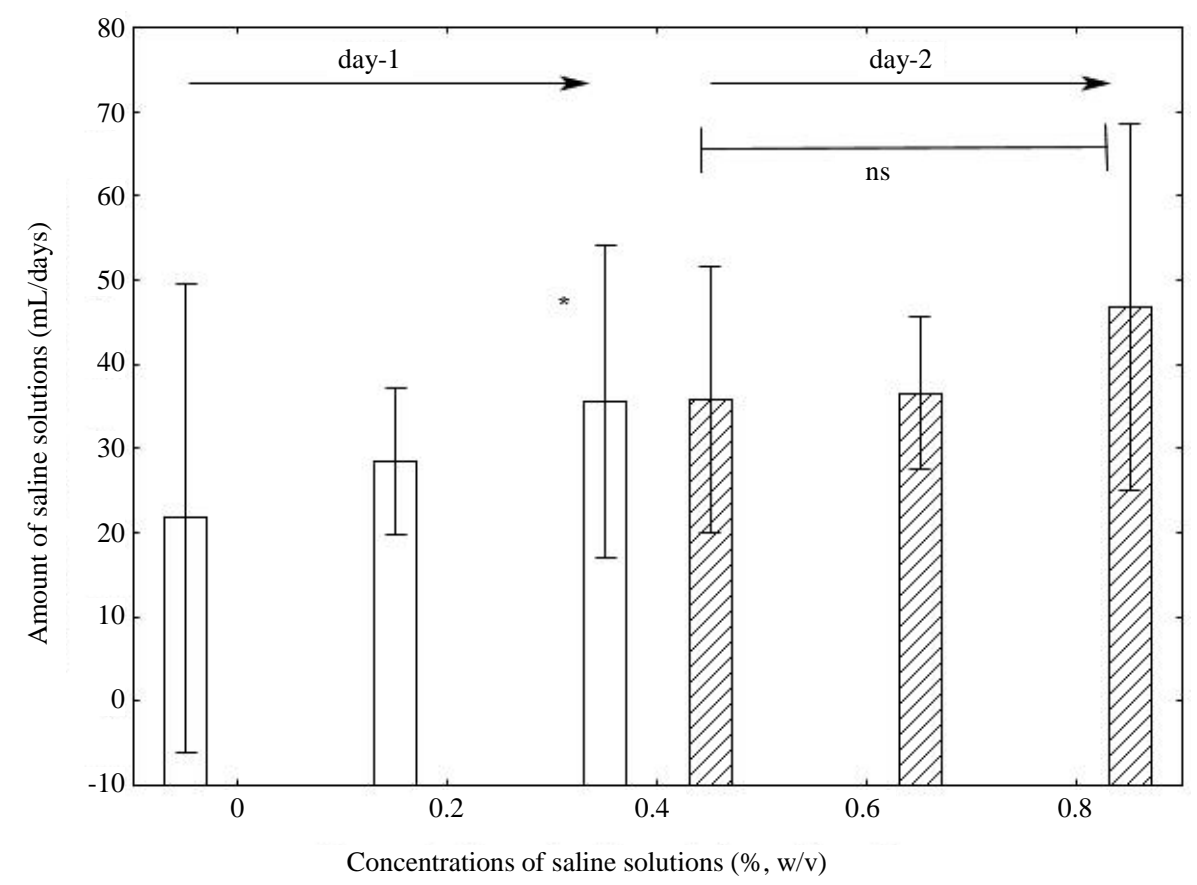

Figure 2. Preliminary study to determine the concentrations of saline solutions for test. To determine the concentrations of saline solutions to be used for salt preference test, we preliminarily examined amounts of saline solutions consumed per day using five 7-week-old Dahl $\mathrm{S}$ rats fed the regular chow. On Day- 1 , the rats consumed greater amounts of $0.4 \%$ saline solution than plain water. On Day-2 there was no group difference among $0.4 \%, 0.6 \%$ and $0.8 \%$ saline solutions. ${ }^{*} \mathrm{p}<0.05$ vs plain water $(0 \%$ saline solution). ns represents statistically not significant.

Table 1. Body weight and systolic blood pressure of the baby rats.

\begin{tabular}{ccccc}
\hline Experiment & Gender of babies & Salt-intake of mothers & BW (grams) & SBP (mmHg) \\
\hline \multirow{2}{*}{ Gestation } & Low-salt & High-salt & $103 \pm 3^{*}$ & $137 \pm 12$ \\
& & Low-salt & $113 \pm 7$ & $143 \pm 8$ \\
& Female & High-salt & $100 \pm 4^{*}$ & $137 \pm 8$ \\
& Male & Low-salt & $112 \pm 8$ & $136 \pm 9$ \\
& & High-salt & $106 \pm 10$ & $132 \pm 5$ \\
Lactation & Lemale & Low-salt & $101 \pm 7$ & $131 \pm 9$ \\
& & High-salt & $96 \pm 10$ & $130 \pm 7$ \\
\hline
\end{tabular}

The values are expressed as means $\pm \mathrm{SD} .{ }^{*} \mathrm{p}<0.001$ vs. high-salt mothers; BW, body weight; SBP, systolic blood pressure.

gestation. In systolic blood pressure, there were no differences between the offspring from low-salt and high-salt mothers.

The salt preference in the male Dahl S rats from low-salt and high-salt mothers during gestation was demonstrated in Figure 3(a). The male baby rats from low-salt and high-salt mothers consumed almost equally any concentration of saline solutions. However, the amounts of saline solutions consumed for 4 days were greater in the male offspring of low-salt mothers than those of high-salt mothers. When we estimated the cumulative amounts of salt intake during the 4-day test period, the salt intake was much greater in the offspring of low-salt mothers than those of high-salt mothers (Figure 3(b)). 


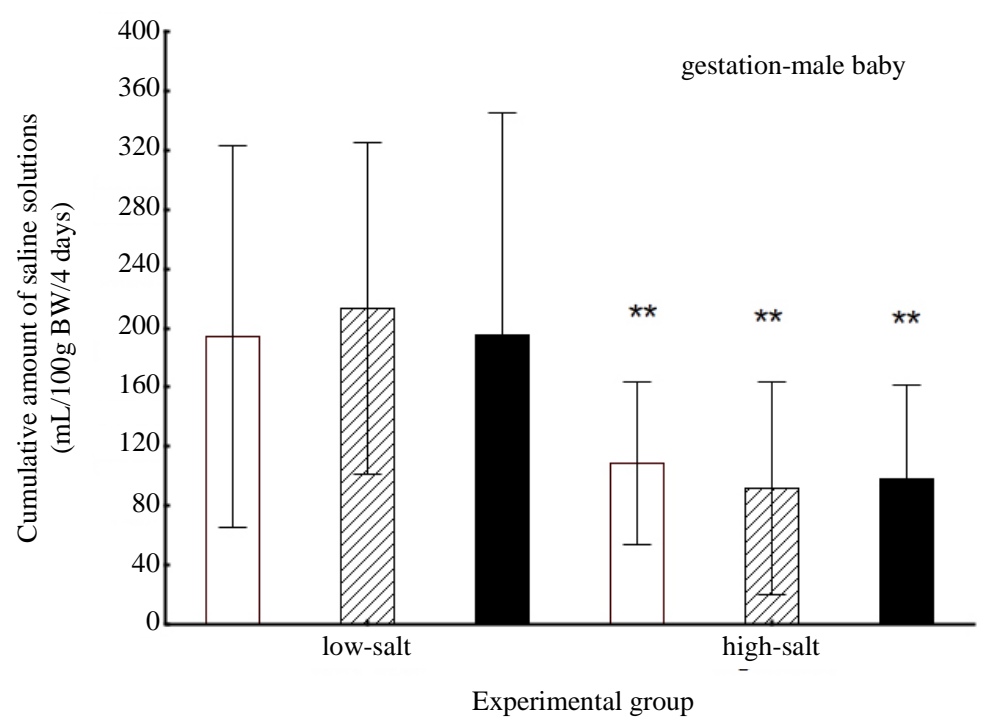

(a)

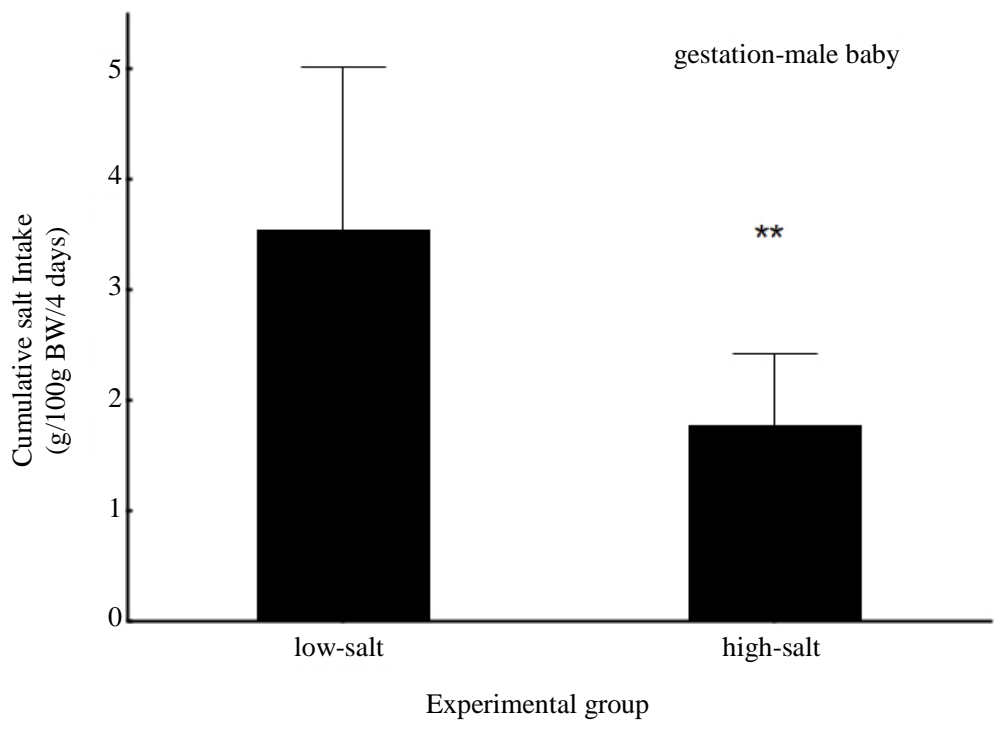

(b)

Figure 3. Amounts of saline solutions in the male baby rats from low-and high-salt mothers during gestation. Salt preference of male baby Dahl S rats from low-salt (left 3 columns, $n=8$ ) and high-salt mothers (right 3 columns, $n$ $=10$ ) during gestation (Figure 3(a)). Open bar, 0.4\% saline solution; shaded bar, $0.6 \%$ saline solution; and solid bar, $0.8 \%$ saline solution. Cumulative salt intake for the 4-day salt preference test was presented in Figure 3(b). ${ }^{* *} \mathrm{p}<$ 0.005 vs the respective values of low-salt mothers.

Similarly, we demonstrated the salt preference in the female offspring of low-salt and high-salt mothers during gestation (Figure 4(a)). The female baby rats from low-salt and high-salt mothers consumed almost equally any concentration of saline solutions. However, the amounts of saline solutions consumed for 4 days were greater in the offspring of low-salt than those of high-salt mothers. Moreover, the amounts of salt intake during the 4-day test period were much greater in the offspring of low-salt mothers than those of high-salt mothers (Figure 4(b)).

In the present study, the values were standardized by 100 grams of body weight. Even when we analyzed the data expressed as per rat, however, we obtained the same results as those obtained from the standardized data. 


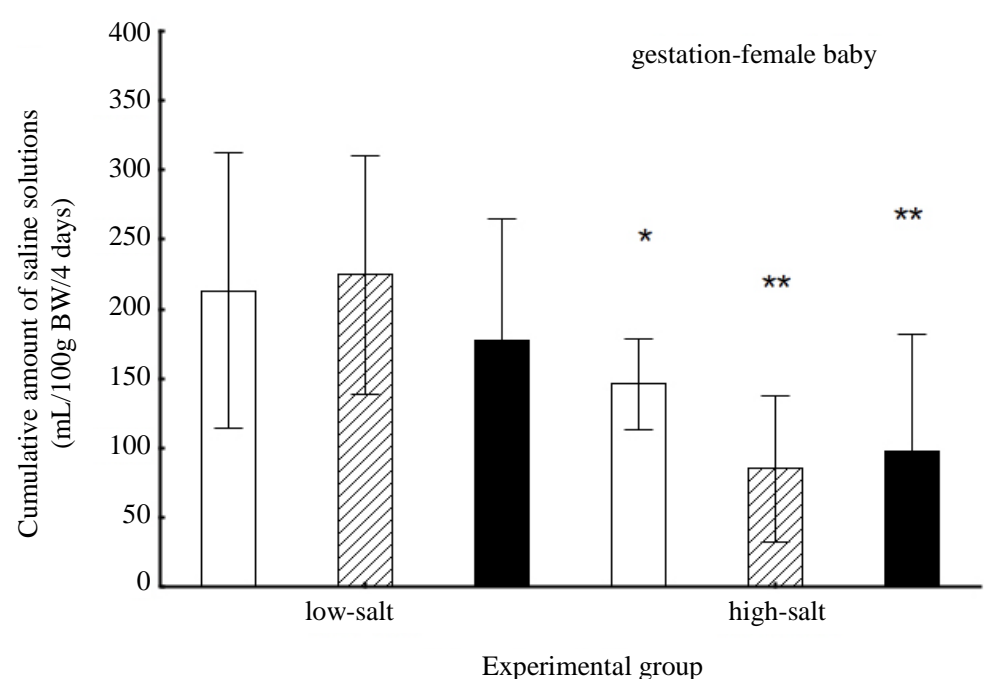

(a)

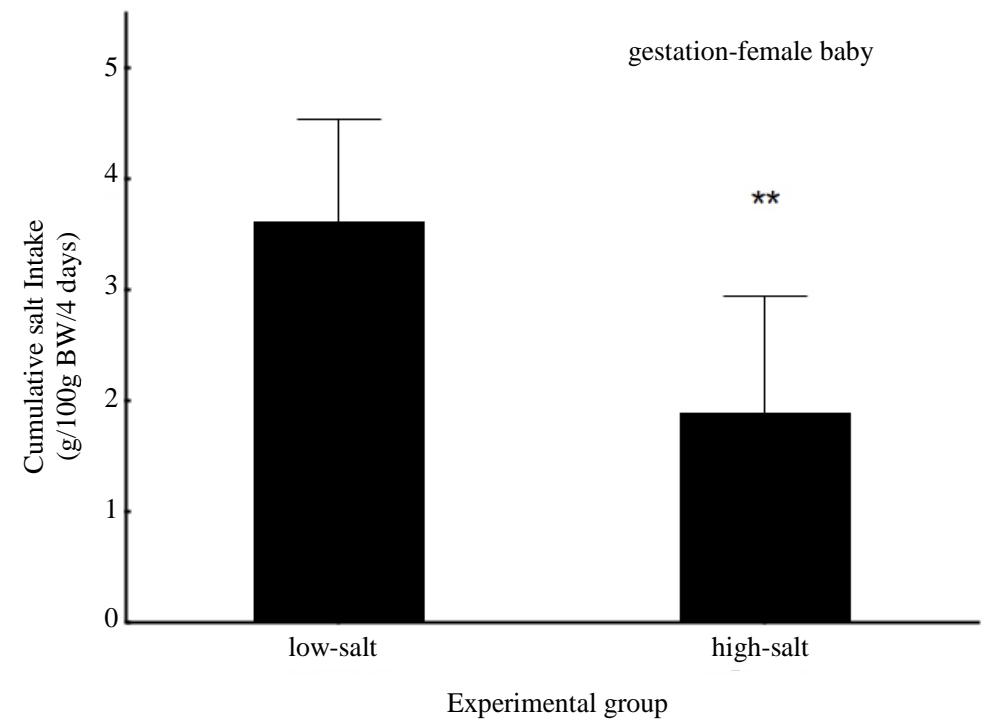

(b)

Figure 4. Amounts of saline solutions and salt intake in the female offspring from low-and high-salt mothers during gestation. Salt preference of female baby Dahl S rats from low-salt (left 3 columns, $\mathrm{n}=6$ ) and high-salt mothers (right 3 columns, $n=6$ ) during gestation (Figure $4(a)$ ). Open bar, $0.4 \%$ saline solution; shaded bar, $0.6 \%$ saline solution; and solid bar, $0.8 \%$ saline solution. Cumulative salt intake for the 4-day salt preference test was presented in Figure 4 (b). ${ }^{*} \mathrm{p}<0.05,{ }^{* * *} \mathrm{p}<0.005$ vs the respective values of low-salt mothers.

\section{2. (EXP-2) Salt Preference of Baby Rats from Mothers of Different Salt Intake during Lactation}

The basal data of the weaning rats were shown in Table 1. In both the male and female baby rats, there were no differences in the body weight between the offspring of low-salt and those of high-salt mothers during lactation. Systolic blood pressure did not differ between the male and female offspring of low and high salt mothers.

The male baby rats from low-salt and high-salt mothers during lactation consumed almost equally any concentration of saline solutions. Interestingly, however, there were no differences in the amounts of $0.4 \%$ and $0.6 \%$ saline solutions between the offspring of low-salt and high-salt mothers. Moreover, the babies of high-salt 
mothers during lactation consumed greater amounts of $0.8 \%$ saline solutions than the offspring of low-salt mothers (Figure 5(a)). Thus, the amounts of salt intake were greater in the offspring of high-salt mothers than those of low-salt mothers (Figure 5(b)).

The salt preference in the female baby rats from low and high salt mothers during lactation was demonstrated in Figure 6(a). The female offspring of low-salt mothers preferred the lower concentrations of saline solutions as indicated by the left three columns, and consumed less $0.6 \%$ saline solutions than the offspring of high-salt mothers. Such a trend of salt preference caused a significant increase in salt intake in the offspring of high-salt mothers compared with those of low-salt mothers during lactation (Figure 6(b)).

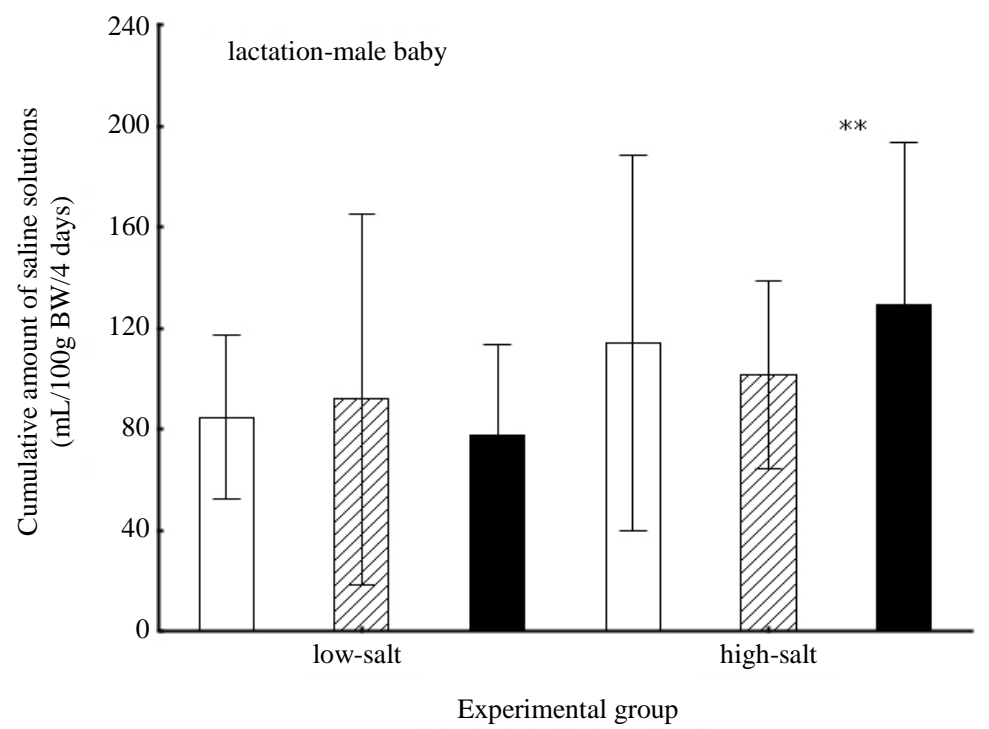

(a)

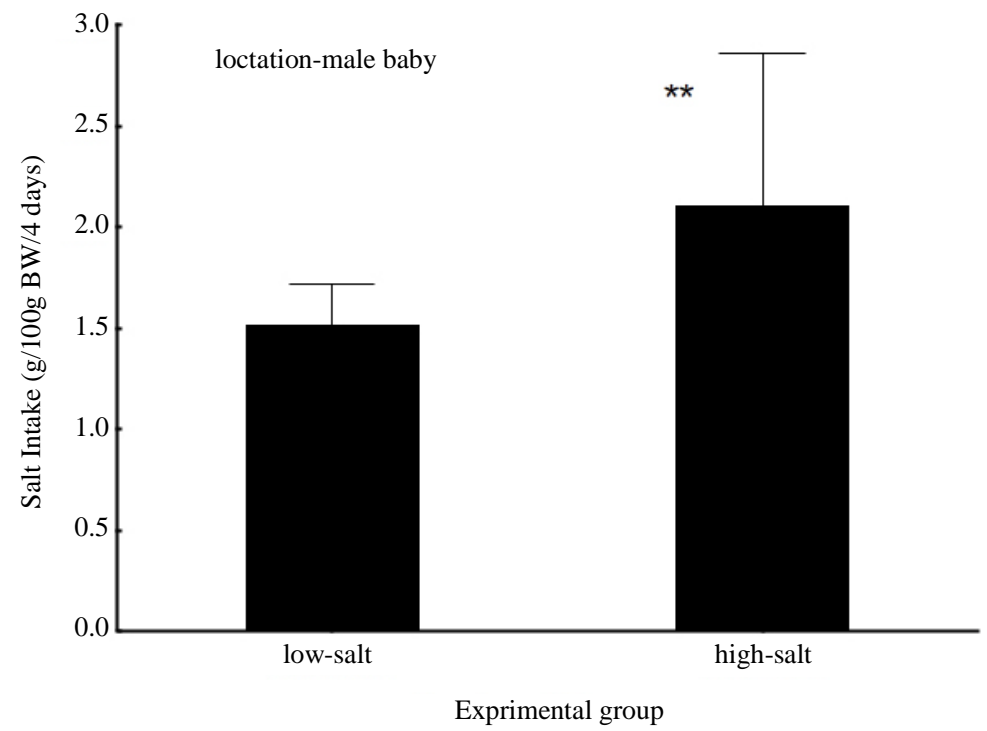

(b)

Figure 5. Amounts of saline solutions and salt intake in the male offspring from low-and high-salt mothers during lactation. Salt preference of male baby Dahl S rats from low-salt (left 3 columns, $n=6$ ) and high-salt mothers during lactation (right 3 columns, $n=6$ ) (Figure 5(a)). Open bar, 0.4\% saline solution; shaded bar, $0.6 \%$ saline solution; and solid bar, $0.8 \%$ saline solution. $\mathrm{Cu}-$ mulative salt intake for the 4-day salt preference test was presented in Figure 5(b). ${ }^{* *} \mathrm{p}<0.005$ vs the value of low-salt mothers. 


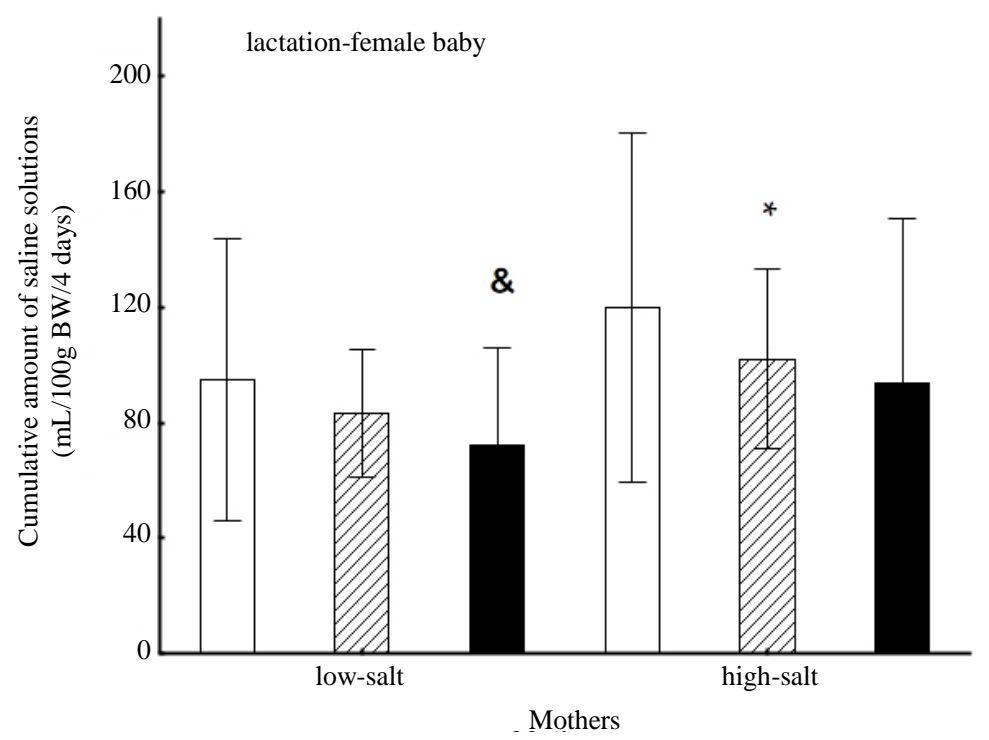

(a)

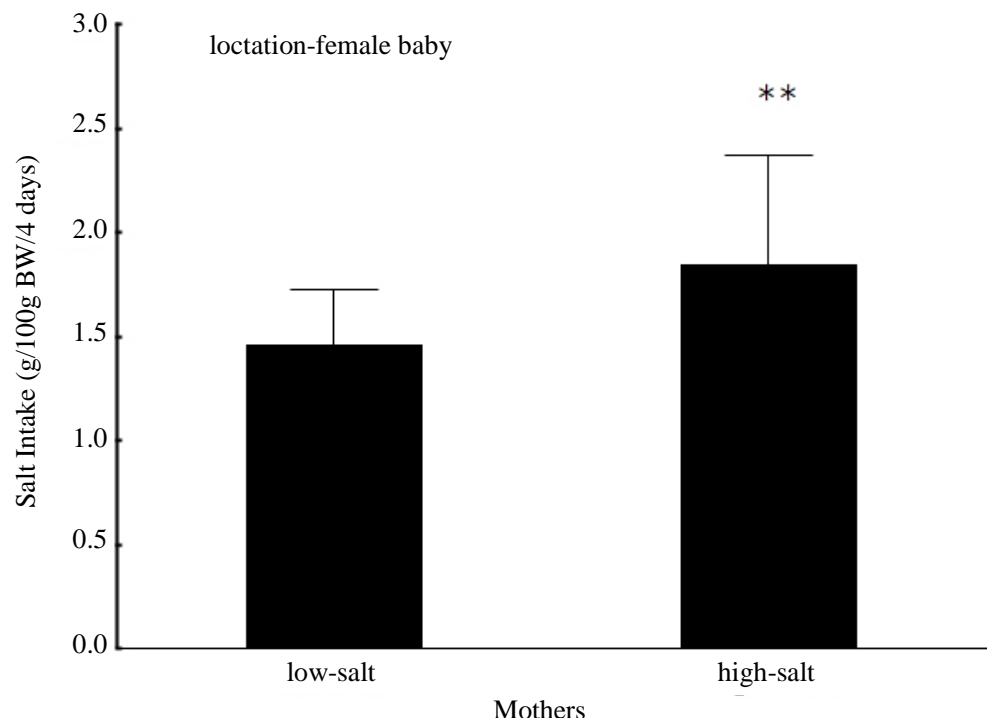

(b)

Figure 6. Amounts of saline solutions and salt intake in the female offspring from low-and high-salt mothers during lactation. Salt preference of female baby Dahl S rats from low-salt (left 3 columns, $n=6$ ) and high-salt mothers during lactation (right 3 columns, $n=6$ ) (Figure 6(a)). Open bar, $0.4 \%$ saline solution; shaded bar, $0.6 \%$ saline solution; and solid bar, $0.8 \%$ saline solution. Cumulative salt intake for the 4-day salt preference test was presented in Figure 6 (b). ${ }^{*} \mathrm{p}<0.05,{ }^{* *} \mathrm{p}<0.005$ vs the values in low-salt mothers. ${ }^{*} \mathrm{p}<$ 0.05 vs $0.4 \%$ saline solution of low-salt mothers.

\section{Discussion}

In the present study, we clearly demonstrated that salt appetite of baby rats was influenced by salt intake of their mothers during gestation and lactation periods. To our surprise, low salt intake in pregnant mothers was associated with an increase of salt intake of their offspring. This was quite different from the influence of high salt diet during lactation. In that case, the baby rats of high-salt mother consumed more salt than those from low-salt mothers. This was seemingly in accordance with our previous data that a high salt diet immediately after weaning was associated with high salt intake in subsequent weeks (unpublished data). Thus, it was suggested that the 
influence of salt intake of mothers in salt preference of their offspring differed depending on the growth stage when they were subject to salt stress. Low salt circumstance seemed critical for intrauterine fetuses to increase their salt appetite after birth.

It is not clear the reason why salt intake increased in the offspring of low-salt mothers during gestation. The baby rats from low and high salt mothers consumed almost equally any concentration of saline solutions. This suggested the increased salt intake was not due to a change of salty taste, but due to a change of salt appetite. Saline solutions increase osmolality of circulating blood, and this would enhance behavior of water-drinking. The baby rats of low-salt mothers conceivably have dysfunction of osmolality homeostasis. In fact, it is well known that angiotensin II (ATII) in the central nervous system enhances water appetite [11]-[13]. Moreover, we have reported that angiotensinogen gene polymorphism (rs 699) is associated with changes of food behavior in young, non-obese female subjects [14]. Based these data, alteration of RAS presumably mediates salt preference in the baby rats from low-salt mothers during gestation. The intrauterine growth state is very critical to program food preference responsible for metabolic alterations in adulthood. The programming seemed quite interesting; however, we could not address its gene-mediated mechanisms at this particular time. We need to design additional studiers including investigation of various humoral factors.

In this context, some investigators from other laboratories reported the relationship between low salt intake of mothers and metabolic diseases of their offspring in adulthood. In 1990, Crystal et al. demonstrated that morning sickness and salt-loss of mothers was associated with infant salt preference [15]-[18]. Salt preference in adulthood might be predicted by salt-loss in infant [19]. More interestingly, salt-restriction is associated with insulin resistance and dyslipidemia in adult Wistar rats [4] [5] [20] [21]. Our data, being in accordance with these studies, demonstrated the importance of intrauterine circumstance in salt preference in infants after weaning.

We also demonstrated that high salt diet during lactation was associated with an increase in salt intake of their offspring. In our preliminary study we found that Dahl S rats fed a high salt diet immediately after weaning exhibited salt preference thereafter (unpublished data). The mechanism and its implications remained to be elucidated. Such salt preference may be related to a change of salty taste, and this is in accordance with the hypothesis that the taste is formed in very early growth stage.

In the present study, the baby rats were fed the regular chow for the test period. We did not determine the amounts of diet consumed during the test period. In our previous study, however, we found that rats weighing $100 \mathrm{~g}$ consumed not more than 20 grams per day, suggesting that 0.6 grams for 4 days. This was much less than the salt intake from saline solutions, and the salt intake from diets unlikely influenced the findings on the salt preference of saline solutions in the present study.

The data obtained in the present study seemed very impressive. However, we need much care to extrapolate this animal study to humans. We move forward to examine the relationship between salt stress of mothers and food preference of their offspring in humans. If this is true, restriction of salt intake particularly during gestation should be careful. On the other hand, during lactation and probably after weaning, the babies might be better restricted to salt intake.

\section{Conclusion}

In the present study, we demonstrated that maternal low salt intake during pregnancy is associated with salt preference in weaning Dahl salt-sensitive rats. Maternal food habit and life-style is influential to determine foodpreference and subsequent hypertensive or metabolic disorders in their offspring. We need further studies to know whether such salt preference in the offspring of low-salt pregnant mothers is related to susceptibility to salt hypertension in Dahl salt sensitive rats.

\section{Declaration of Interest}

The authors report no conflicts of interest. The authors alone are responsible for the content and writing of the paper.

\section{Acknowledgements}

The authors acknowledge Grant-in-Aids from Center for Interdisciplinary Studies of Science and Culture, Kyoritsu Women's University \& Junior College. 


\section{References}

[1] Uiterwaal, C.S., Anthony, S., Launer, L.J., Witteman, J.C., Trouwborst, A.M., Hofman, A. and Grobbee, D.E. (1997) Birth Weight, Growth, and Blood Pressure: An Annual Follow-Up Study of Children Aged 5 through 21 Years. Hypertension, 30, 267-271.

[2] Johansson, S., Norman, M., Legnevall, L., Dalmaz, Y., Lagercrantz, H. and Vanpée, M. (2007) Increased Catecholamines and Heart Rate in Children with Low Birth Weight: Perinatal Contributions to Sympathoadrenal Overactivity. Journal of Internal Medicine, 261, 480-487.

[3] Dalziel, S.R., Parag, V., Rodgers, A. and Harding, J.E. (2007) Cardiovascular Risk Factors at Age 30 Following PreTerm Birth. International Journal of Epidemiology, 36, 907-915.

[4] Hovi, P., Andersson, S., Eriksso, J.G., Järvenpää, A.L., Strang-Karlsson, S., Mäkitie, O. and Kajantie, E. (2007) Glucose Regulation in Young Adults with Very Low Birth Weight. The New England Journal of Medicine, 356, $2053-2063$.

[5] Rotteveel, J., van Weissenbruch, M.M., Twisk, J.W. and Delemarre-Van de Waal, H.A. (2008) Infant and Childhood Growth Patterns, Insulin Sensitivity, and Blood Pressure in Prematurely Born Young Adults. Pediatrics, 122, 313-321.

[6] Piecha, G., Koleganova, N., Ritz, E., Müller, A., Fedorova, O.V., Bagrov, A.Y., Lutz, D., Schirmacher, P. and GrossWeissmann, M.L. (2012) High Salt Intake Causes Adverse Fetal Programming Vascular Effects beyond Blood Pressure. Nephrology Dialysis Transplantation, 27, 3464-3476.

[7] Alves-Rodrigues, E.N., Veras, M.M., Rosa, K.T., de Castro, I., Furukawa, L.N., Oliveira, I.B., Souza, R.M. and Heimann, J.C. (2012) Salt Intake during Pregnancy Alters Offspring’s Myocardial Structure. Nutrition, Metabolism Cardiovascular Diseases, 23, 481-486.

[8] Porter, J.P., King, S.H. and Honeycutt, A.D. (2007) Prenatal High-Salt Diet in the Sprague-Dawley Rat Programs Blood Pressure and Heart Rate Hyperresponsiveness to Stress in Adult Female Offspring. American Journal of Physiology. Regulatory, Integrative and Comparative Physiology, 293, R334-R342.

[9] Crystal, S.R. and Bernstein, I.L. (1995) Morning Sickness: Impact on Offspring Salt Preference. Appetite, 25, $231-240$.

[10] Friedman, M. and Freed, S.C. (1949) Microphonic Manometer for Indirect Determination of Systolic Blood Pressure in the Rat. Proceedings of the Society for Experimental Biology and Medicine, 70, 670-672.

[11] Yang, W., Mao, C., Xia, F., Zheng, J., Wang, A., Zhu, L., He, R. and Xu, Z. (2010) Changed Salt Appetite and Central Angiotensin II-Induced Cellular Activation in Rat Offspring Following Hypoxia during Fetal Stages. Peptides, 31, 1177-1183. http://dx.doi.org/10.1016/j.peptides.2010.03.009

[12] Nakano-Tateno, T., Shichiri, M., Suzuki-Kemuriyama, N., Tani, Y., Izumiyama, H. and Hirata, Y. (2012) Prolonged Effects of Intracerebroventricular Angiotensin II on Drinking, Eating and Locomotor Behavior in Mice. Regulatory Peptides, 173, 86-92. http://dx.doi.org/10.1016/j.regpep.2011.09.011

[13] Mecawi, A.S., Araujo, I.G., Rocha, F.F., Coimbra, T.M., Antunes-Rodrigues, J. and Reis, L.C. (2010) Ontogenetic Role of Angiontensin-Converting Enzyme in Rats: Thirst and Sodium Appetite Evaluation. Physiology \& Behavior, 99, 118-124. http://dx.doi.org/10.1016/j.physbeh.2009.10.018

[14] Sasaki, M., Yamada, K., Namba, H., Yoshinaga, M., Du, D. and Uehara, Y. (2013) Angiotensinogen Gene Polymorphisms and Food-intake Behavior in Young, Normal Female Subjects in Japan. Nutrition, 29, 60-65.

[15] Crystal, S.R., Bowen, D.J. and Bernstein, I.L. (1999) Morning Sickness and Salt Intake, Food Cravings, and Food Aversions. Physiology \& Behavior, 67, 181-187. http://dx.doi.org/10.1016/S0031-9384(99)00055-4

[16] Crystal, S.R. and Bernstein, I.L. (1998) Infant Salt Preference and Mother’s Morning Sickness. Appetite, 30, $297-307$. http://dx.doi.org/10.1006/appe.1997.0144

[17] Nicolaidis, S., Galaverna, O. and Metzler, C.H. (1990) Extracellular Dehydration during Pregnancy Increases Salt Appetite of Offspring. American Journal of Physiology, 258, R281-R283.

[18] Galaverna, O., Nicolaïdis, S., Yao, S.Z., Sakai, R.R. and Epstein, A.N. (1995) Endocrine Consequences of Prenatal Sodium Depletion Prepare Rats for High Need-Free NaCl Intake in Adulthood. American Journal of Physiology, 269, R578-R583.

[19] Leshem, M. (1998) Salt Preference in Adolescence Is Predicted by Common Prenatal and Infantile Mineralofluid Loss. Physiology \& Behavior, 63, 699-704. http://dx.doi.org/10.1016/S0031-9384(97)00525-8

[20] Vidonho Jr., A.F., Silva, A.A., Catanozi, S., Rocha, J.C., Beutel, A., Carillo, B.A., Furukawa, L.N., Campos, R.R., Bergamaschi, C.M., Carpinelli, A.R., Quintão, E.C., Dolnikoff, M.S. and Heimann, J.C. (2004) Perinatal Salt Restriction: A New Pathway to Programming Insulin Resistance and Dyslipidemia in Adult Wistar Rats. Pediatric Research, 56, 842-848. http://dx.doi.org/10.1203/01.PDR.0000145258.75160.5B

[21] Lopes, K.L., Furukawa, L.N., de Oliveira, I.B., Dolnikoff, M.S. and Heimann, J.C. (2008) Perinatal Salt Restriction: A New Pathway to Programming Adiposity Indices in Adult Female Wistar Rats. Life Sciences, 82, 728-732.

http://dx.doi.org/10.1016/j.lfs.2008.01.003 
Scientific Research Publishing (SCIRP) is one of the largest Open Access journal publishers. It is currently publishing more than 200 open access, online, peer-reviewed journals covering a wide range of academic disciplines. SCIRP serves the worldwide academic communities and contributes to the progress and application of science with its publication.

Other selected journals from SCIRP are listed as below. Submit your manuscript to us via either submit@scirp.org or Online Submission Portal.
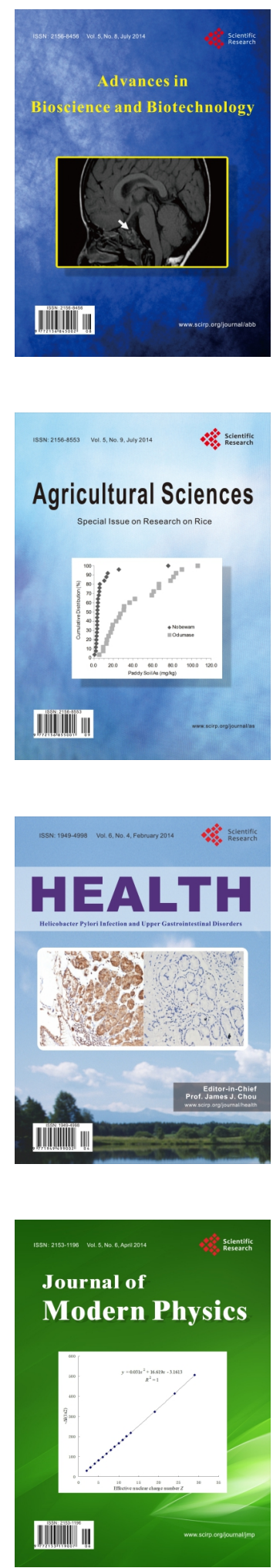
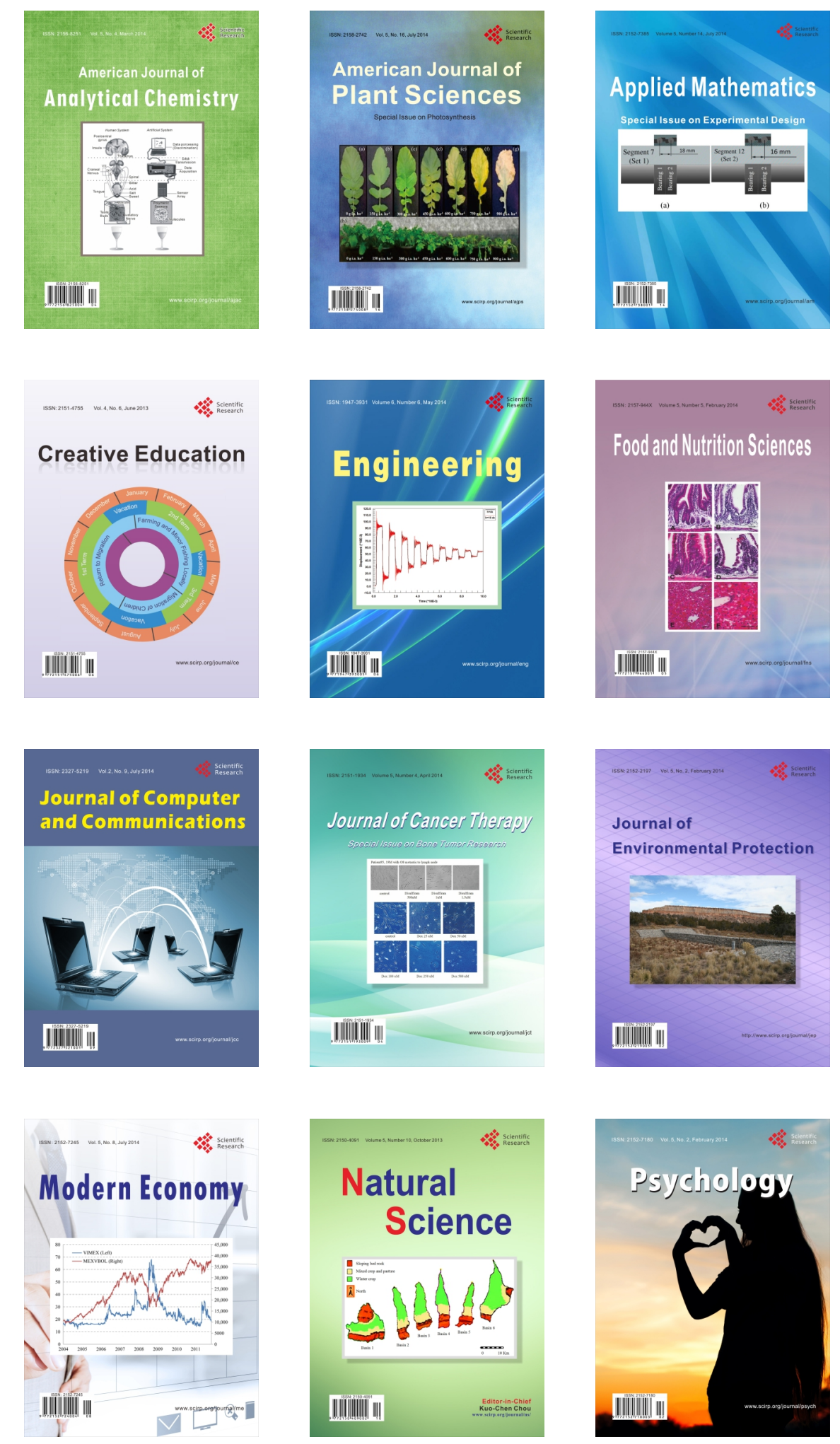